\title{
Composite vectors in Higgless models at the LHC
}

\section{Antonio Enrique Cárcamo Hernández ${ }^{* \dagger}$}

Scuola Normale Superiore Di Pisa and INFN, Sezione di Pisa, Pisa, Italy

E-mail: antonio.carcamo@sns.it

In the context of a strongly coupled Electroweak Symmetry Breaking, composite triplet of heavy vectors degenerate in mass belonging to the $S U(2)_{L+R}$ adjoint representation may arise from a new strong interaction invariant under the global $S U(2)_{L} \times S U(2)_{R}$ symmetry, which is spontaneously broken down to $S U(2)_{L+R}$. Assuming that the interactions among these heavy vectors states and with the Standard Model gauge bosons are described by an $S U(2)_{L} \times$ $S U(2)_{R} / S U(2)_{L+R}$ Effective Chiral Lagrangian, the heavy vector pair production at the LHC by Vector Boson Fusion and Drell-Yan annihilation is studied in this framework. The expected rates of same sign di-lepton and tri-lepton events from the decay of the composite vectors into Standard Model gauge bosons are computed. The determination of the corresponding SM backgrounds is deferred for a future work.

XVIII International Workshop on Deep-Inelastic Scattering and Related Subjects April 19 -23, 2010

Convitto della Calza, Firenze, Italy

* Speaker.

$\dagger$ A footnote may follow. 


\section{Introduction}

It is known that there are two pictures used to explain the mechanism of Electroweak Symmetry Breaking, that is, weakly and strongly coupled. The LHC is going to determine which of these pictures is realized by nature. A weakly coupled dynamics describing the mechanism of the Electroweak Symmetry Breaking (EWSB) is provided by the Standard Model and its Supersymmetric extensions. In the Standard Model, the existence of one zero spin and neutral scalar particle called the Higgs boson is assumed in order to explain the generation of all the fermions and bosons masses through the Yukawa couplings to fermions. The Higgs boson is also important in keeping under control the unitarity for the elastic and inelastic channels of the gauge boson scattering and is useful in extrapolating the model at weak coupling down to the Planck scale. A light Higgs boson can sucessfully account for the Electroweak Precision Tests. In spite of the very good agreement of the Standard Model predictions with experimental data, the Higgs boson has not been found and then, the mechanism of Electroweak Symmetry Breaking responsible for the generation of the masses of the fermions and bosons remains to be explained. Besides that, the Standard Model has the so called hierarchy problem, which is the strong sensitivity of the Higgs mass to very short distance scales. These problems provide plausible motivations for considering strongly coupled theories of EWSB, which become non-perturbative above the Fermi scale and the breaking is achieved through some condensate. In the most general framework of a strongly interacting dynamics for EWSB, one can have composite resonances which could be spin- 0 , spin- $1 / 2$ and spin- 1 states. These composite particles are bound states of more fundamental constituents which are held together by a new strong interaction. Here the case in which the strong dynamics responsible for EWSB gives rise to a triplet of composite vectors belonging to the adjoint representation of the custodial symmetry group $S U(2)_{L+R}$ is considered. These composite vectors states are very important in keeping under control the longitudinal SM gauge boson scattering up to a cutoff $\Lambda \simeq 3 \mathrm{TeV}$ and under suitable conditions can account for Electroweak Precision Tests [1]. This work is devoted to the study of the heavy vectors pair production at the LHC by Vector Boson Fusion and Drell Yan annihilation.

\section{Effective Chiral Lagrangian with massive spin one fields.}

In the framework of a strongly interacting dynamics for the EWSB, the interactions among the composite triplet heavy vectors, composite fermions and the SM particles can be described by the following model independent $S U(2)_{L} \times S U(2)_{R} / S U(2)_{L+R}$ Chiral Lagrangian invariant under parity [2]:

$$
\begin{aligned}
\mathscr{L}= & \frac{v^{2}}{4}\left\langle D_{\mu} U\left(D^{\mu} U\right)^{\dagger}\right\rangle-\frac{1}{2 g^{2}}\left\langle W_{\mu v} W^{\mu v}\right\rangle-\frac{1}{2 g^{\prime 2}}\left\langle B_{\mu v} B^{\mu v}\right\rangle-\frac{1}{4}\left\langle\hat{V}^{\mu v} \hat{V}_{\mu v}\right\rangle+\frac{M_{V}^{2}}{2}\left\langle V^{\mu} V_{\mu}\right\rangle \\
& -\frac{i g_{V}}{2 \sqrt{2}}\left\langle\hat{V}^{\mu v}\left[u_{\mu}, u_{v}\right]\right\rangle-\frac{f_{V}}{2 \sqrt{2}}\left\langle\hat{V}^{\mu v}\left(u W_{\mu v} u^{\dagger}+u^{\dagger} B_{\mu v} u\right)\right\rangle+\frac{i g_{K}}{2 \sqrt{2}}\left\langle\hat{V}_{\mu v} V^{\mu} V^{v}\right\rangle \\
& +g_{1}\left\langle V_{\mu} V^{\mu} u^{\alpha} u_{\alpha}\right\rangle+g_{2}\left\langle V_{\mu} u^{\alpha} V^{\mu} u_{\alpha}\right\rangle+g_{3}\left\langle V_{\mu} V_{v}\left[u^{\mu}, u^{v}\right]\right\rangle+g_{4}\left\langle V_{\mu} V_{v}\left\{u^{\mu}, u^{v}\right\}\right\rangle \\
& +g_{5}\left\langle V_{\mu}\left(u^{\mu} V_{v} u^{v}+u^{v} V_{v} u^{\mu}\right)\right\rangle+i g_{6}\left\langle V_{\mu} V_{v}\left(u W^{\mu v} u^{\dagger}+u^{\dagger} B^{\mu v} u\right)\right\rangle
\end{aligned}
$$

where $f_{V}=\frac{F_{V}}{M_{V}}, g_{V}=\frac{G_{V}}{M_{V}}, g_{K}, g_{i}$ with $i=1,2, \cdots, 6$ are dimensionless parameters and the following 
relations are satisfied:

$$
\begin{aligned}
& U(x)=e^{i \hat{\pi}(x) / v}, \quad \hat{\pi}(x)=\tau^{a} \pi^{a}=\left(\begin{array}{cc}
\pi^{0} & \sqrt{2} \pi^{+} \\
\sqrt{2} \pi^{-} & -\pi^{0}
\end{array}\right), \\
& D_{\mu} U=\partial_{\mu} U-i B_{\mu} U+i U W_{\mu}, \quad W_{\mu}=\frac{g}{2} \tau^{a} W_{\mu}^{a}, \quad B_{\mu}=\frac{g^{\prime}}{2} \tau^{3} B_{\mu}^{0}, \\
& W_{\mu v}=\partial_{\mu} W_{v}-\partial_{\nu} W_{\mu}-i\left[W_{\mu}, W_{v}\right], \quad B_{\mu \nu}=\partial_{\mu} B_{v}-\partial_{\nu} B_{\mu}
\end{aligned}
$$

The field strength tensor $\hat{V}_{\mu \nu}=\nabla_{\mu} V_{v}-\nabla_{\nu} V_{\mu}$ is written in terms of the $S U(2)_{L} \times S U(2)_{R}$ covariant derivative

$$
\nabla_{\mu} V_{v}=\partial_{\mu} V_{v}+\left[\Gamma_{\mu}, V_{v}\right], \quad V_{\mu}=\frac{1}{\sqrt{2}} V_{\mu}^{a} \tau^{a}
$$

with the connection $\Gamma_{\mu}$ given by

$$
\Gamma_{\mu}=\frac{1}{2}\left[u^{\dagger}\left(\partial_{\mu}-i B_{\mu}\right) u+u\left(\partial_{\mu}-i W_{\mu}\right) u^{\dagger}\right], \quad u \equiv \sqrt{U}, \quad \Gamma_{\mu}^{\dagger}=-\Gamma_{\mu} .
$$

and $u_{\mu}=u_{\mu}^{\dagger}=i u^{\dagger} D_{\mu} U u^{\dagger}$.

Here the following assumptions have been made:

1. Before weak gauging, the Lagrangian responsible for EWSB has a $S U(2)_{L} \times S U(2)_{R}$ global symmetry which is spontaneously broken by the new strong dynamics down to $S U(2)_{L+R}$. The spontaneous breaking of the global symmetry also leads to the breaking of the standard electroweak gauge symmetry, $S U(2)_{L} \times U(1)_{Y}$, down to the electromagnetic $U(1)$.

2. The strong dynamics produces only one vector triplet of vectors $V_{\mu}^{a}$ belonging to the adjoint representation of $S U(2)_{L+R}$ custodial group. These vector states are mass degenerate and their mass is below the cut-off $\Lambda \approx 3 \mathrm{TeV}$. The parity odd heavy vectors are integrated out. The new $V$ states couples to fermions only via SM gauge interactions.

\section{Gauge model scenario.}

The scattering amplitudes for the processes $W_{L} W_{L} \rightarrow V_{L} V_{L}$ and $W_{L} W_{L} \rightarrow V_{L} V_{T}$ will grow at most as $\frac{s}{v^{2}}$ and the $q \bar{q} \rightarrow V V$ scattering amplitude will go as a constant only when [2]:

$$
g_{K}=\frac{1}{g_{V}}, \quad f_{V}=2 g_{V}, \quad g_{1}=g_{2}=g_{4}=g_{5}=0, \quad g_{3}=-\frac{1}{4}, \quad g_{6}=\frac{1}{2}
$$

This choice of parameters defines the Gauge Model Scenario, which corresponds to the case in which the new heavy vector states are the gauge vectors arising from a spontaneously broken symmetry $G=S U(2)_{L} \otimes S U(2)_{R} \otimes S U(2)_{C}$ to the diagonal subgroup $S U(2)_{L+R+C}$ by non linear sigma model [2]. The asymptotic behaviour of the $W_{L} W_{L} \rightarrow V_{L} V_{L}$ and $W_{L} W_{L} \rightarrow V_{L} V_{T}$ can be improved by the addition of a composite scalar as discussed in [3].

\section{Vector Pair production cross sections by Vector Boson Fusion.}

The total cross sections at the LHC for heavy vector pair production via Vector Boson Fusion (VBF) in the gauge and composite model scenario are respectively shown in Figures 1.a and 1.b. 


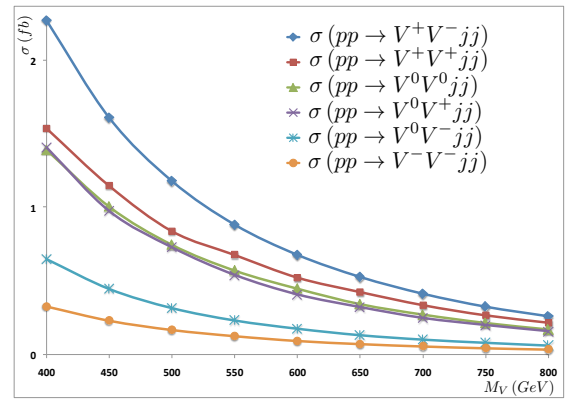

(1.a)

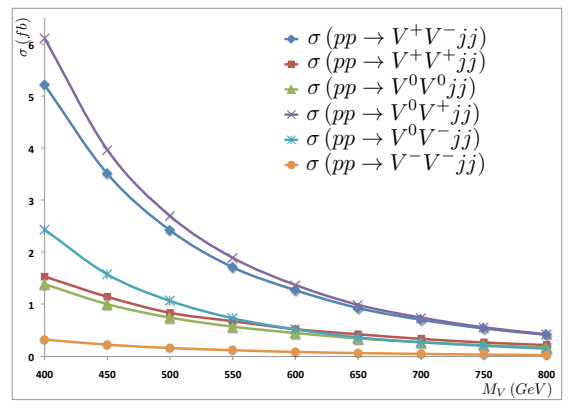

(1.b)

Figure 1: Total cross sections for Vector pair production via Vector Boson fusion in a Gauge Model (1.a) and in a Composite Model (1.b) [2]

The heavy vector mass is taken to range from $400 \mathrm{GeV}$ to $800 \mathrm{GeV}$. The coupling $G_{V}$ of the heavy vector to two longitudinal Standard Model Gauge bosons is taken to be $G_{V}=200 \mathrm{GeV}$, which is in the allowed region consistent with the unitarity constraint in the elastic channel for longitudinal SM gauge boson scattering [1]. In the composite model scenario, all the parameters are kept as in the gauge model with the exception of $g_{K} g_{V}=1 / \sqrt{2}$. These total cross sections have been computed by using the Matrix Element Generator CalcHEP and setting the acceptance cuts $p_{T}>30 \mathrm{GeV}$ and $|\eta|<5$ for the forward quark jets. These cross sections, which involve all polarizations of the intermediate light bosons, have also a weak dependence on $F_{V}$, which is set to zero. Since in the gauge model scenario the growth of the $W W \rightarrow V V$ amplitude is more under control than in the composite model scenario, the total cross section for vector pair production via VBF for the gauge model has lower values than in the composite model, as shown in Figures 1.a and 1.b.

\section{Pair production cross sections by Drell Yan annihilation.}

The total cross sections at the LHC for heavy vector pair production via Drell Yan annihilation as function of the heavy vector mass in the gauge and composite model scenario are respectively shown in Figures 2.a and 2.b. The heavy vector mass is taken to range from $400 \mathrm{GeV}$ to $800 \mathrm{GeV}$. As for the vector boson fusion, $G_{V}=200 \mathrm{GeV}$ while in the composite model scenario the parameters are kept as in the gauge model except for $g_{K} g_{V}=1 / \sqrt{2}$. Since in the gauge model scenario the Drell Yan vector pair production amplitude goes as a constant at high energies, the total cross sections in this case are lower than in the composite model.

\section{Same-sign di-lepton and tri-lepton events at LHC.}

Since the heavy vector have dominant decay mode into pair of SM Gauge bosons with branching ratio very close to one, the vector pair production by VBF and DY will lead to 4 SM gauge bosons in the final state. The following table shows the total number of same sign di-lepton and tri-lepton events ( $e$ or $\mu$ from $W$ decays) at the LHC for a reference integrated luminosity of $\int \mathscr{L} d t=100 \mathrm{fb}^{-1}$. The heavy vector mass is taken to be $M_{V}=500 \mathrm{GeV}$. 


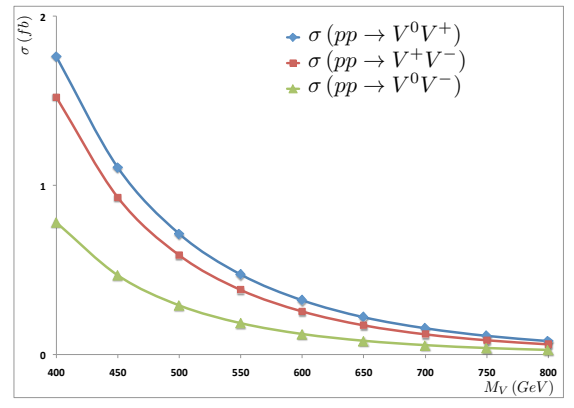

(2.a)

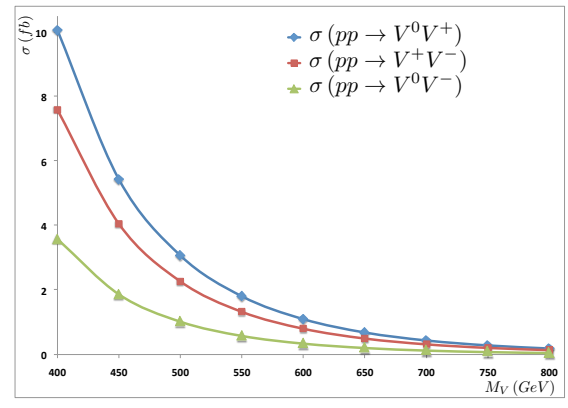

(2.b)

Figure 2: Total cross sections for Vector pair production via Drell-Yan annihilation in a Gauge Model (2.a) and in a Composite Model (2.b) [2]

\begin{tabular}{|c|c|c|}
\hline & di-leptons & tri-leptons \\
\hline Vector Boson Fusion (Gauge Model) & 16 & 3 \\
\hline Drell Yan annihilation (Gauge Model) & 5 & 1 \\
\hline Vector Boson Fusion (Composite Model) & 28 & 6 \\
\hline Drell Yan annihilation (Composite Model) & 18 & 4 \\
\hline
\end{tabular}

Table 1: Number of same sign di-lepton and trilepton events at the LHC

\section{Conclusions}

In the framework of strongly interacting dynamics for EWSB composite heavy vector states may exist and the interactions among themselves and with the Standard Model gauge bosons can be described by a $S U(2)_{L} \times S U(2)_{R} / S U(2)_{L+R}$ Effective Chiral Lagrangian. The total cross sections at the LHC for the vector pair production by Vector Boson Fusion and Drell Yan annihilation are of order of few $f b$. The numbers of same sign Dilepton and Trilepton events at LHC with an integrated luminosity of $100 \mathrm{fb}^{-1}$ are of order of 10 . Further detailed studies will have to be made to assess the detectability of these processes above the Standard Model backgrounds.

\section{Acknowledgements}

This work was done in collaboration with Riccardo Barbieri, Gennaro Corcella, Riccardo Torre and Enrico Trincherini. It is pleasure to thank all of them for this fruitful work. I also thank the organizers of the XVIII International DIS2010 Workshop for inviting me to present this talk.

\section{References}

[1] R. Barbieri, G. Isidori, V. S. Rychkov and E. Trincherini, Phys. Rev. D 78 (2008) 036012 [arXiv:0911.1942[hep-ph]].

[2] R. Barbieri, A. E. Cárcamo Hernández, G. Corcella, R. Torre and E. Trincherini, JHEP 03 (2010) 068 [arXiv:0911.1942[hep-ph]]

[3] A. E. Cárcamo Hernández and R. Torre [arXiv:1005.3809[hep-ph]]

[4] O. Cata, G. Isidori and J. F. Kamenik, Nucl. Phys. B 822 (2009) 230 [arXiv:0905.0490[hep-ph]]. 